\title{
DIRECT SUMS OF COUNTABLY GENERATED MODULES OVER COMPLETE DISCRETE VALUATION RINGS
}

\begin{abstract}
CHANG MO BANG ${ }^{1}$
Abstract. Throughout this paper, $R$ will denote an arbitrary but fixed complete discrete valuation ring. We shall show that two reduced $R$-modules which are direct sums of countably generated $R$-modules are isomorphic if and only if they have the same Ulm invariants and the same basis type. This is a generalization of the celebrated Ulm and Kolettis theorem.
\end{abstract}

1. Introduction. For the sake of convenience, a cardinal shall be viewed as the first ordinal of that cardinality and called a cordinal. For each countable cordinal $k$, define $(c, R, k)$ to be the class of all countably generated reduced $R$-modules having torsion-free rank $\leqq k$ and $D(c, R, k)$ to be that of all direct sums of members of $(c, R, k)$. Clearly, $(c, R, k) \subset D(c, R, k) \subset D(c, R, \omega)$, and each member of $D(c, R, \omega)$ is a reduced direct sum of countably generated $R$-modules. Notice that countable $p$-primary abelian groups are members of $(c, R, 0)$ and their direct sums are members of $D(c, R, 0)$ where $R$ is the ring of $p$-adic integers. Classifications, up to isomorphisms, of all members of $(c, R, 0),(c, R, 1), D(c, R, 0),(c, R$, finite), $(c, R, \omega)$ were obtained by Ulm [12], Kaplansky and Mackey [7], Kolettis [8], Rotman and Yen [11], Bang [1], respectively. In particular, we need to recall the following for later use.

Theorem 1. Let $N, N^{\prime} \in(c, R, \omega)$. Then, $N \simeq N^{\prime}$ if and only if they have the same Ulm invariant and the same height equivalence.

For the proof, see [1]. Our purpose is to classify all members of $D(c, R, \omega)$ by proving the following. This result was announced in part in [2].

Theorem 2. Let $M, M^{\prime} \in D(c, R, \omega)$. Then $M \simeq M^{\prime}$ if and only if they have the same Ulm invariant and the same basis type.

Received by the editors February 26, 1970 and, in revised form, August 4, 1970.

AMS 1969 subject classification. Primary 2030, 2027.

Key words and phrases. Direct sums of countably generated reduced modules, complete discrete valuation ring, Ulm invariant, basis type, height-preserving isomorphism, the Ulm theorem, the Kolettis theorem.

1 The author would like to acknowledge the support of the National Science Foundation Grant GP-8725 and GP-11804.

Copyright (c) 1971, American Mathematical Society 
Clearly, by adding enough zero summands if necessary, we may write for some cordinal $I$

$$
M=\bigoplus\left\{M_{i}: i \in I\right\} \quad \text { and } \quad X^{\prime}=\bigoplus\left\{M_{i}^{\prime}: i \in I\right\}
$$

with all summands $M_{i}, M_{i}^{\prime} \in(c, R, \omega)$. The main idea of the proof is to show that there is a partition of $I$ into countable subsets $\left\{I_{j}\right\}$ such that $\bigoplus\left\{M_{i}: i \in I_{j}\right\}$ and $\bigoplus\left\{M_{i}^{\prime}: i \in I_{j}\right\}$ have the same Ulm invariant and the same height equivalence for each $j$. Then, by Theorem 1 , we have $\bigoplus\left\{M_{i}: i \in I_{j}\right\} \simeq \bigoplus\left\{M_{i}^{\prime}: i \in I_{j}\right\}$ for each $j$ and consequently $M \simeq M^{\prime}$. This will be done through four lemmas.

2. Terminologies. For general notations and terminologies, see [4] and [6]. Throughout this paper, $p$ denotes a fixed prime of $R$. Therefore, $R$ is a complete local principal ideal domain with a maximal ideal $(p)$. Since all primes of $R$ are associates of $p$, the height $h(x)$ of $x$ in this paper practically means the $p$-height $h_{p}(x)$ of $x$. Let $k$ be a cordinal. Let $R^{(k)}$ designate the direct sum of $k$ copies of $R$, $\bigoplus\{R: i<k\}$. Each element $\alpha=\left\{a_{i} \in R: i<k\right\} \in R^{(k)}$ will be viewed as a $k$-dimensional row vector (with only finitely many nonzero coordinates). Let $N$ be an $R$-module having torsion-free rank $k$ (we shall write $r(N)=k$ to indicate the torsion-free rank of $N$ ). Every torsion-free basis (a maximal linearly independent subset) $\eta$ $=\left\{y_{i} \in N: i<k\right\}$ of $N$ will be, on the other hand, viewed as a $k$ dimensional column vector. Notice that the matrix product $\alpha \eta$ $=\sum\left\{a_{i} y_{i}: i<k\right\}$ is an element of $N$ since $\alpha$ is row-finite. Let $m(Q)$ denote the class of all row-finite square matrices over the quotient field $Q$ of $R$. A matrix $\gamma \in M(Q)$ will be said to be integral if all entries of $\gamma$ are elements of $R$. Let $\gamma$ be a $k \times k$ integral matrix of $m(Q)$. Then, the matrix product $\alpha \gamma$ is an element of $R^{(k)}$ and the matrix product $\alpha \gamma \eta$ is an element of $N$ (the associativity holds). For each subset $A \subseteq N,[A]$ designates the submodule of $N$ generated by $A$, and $N_{t}$ the torsion part of $N$. Hereafter, torsion-free basis will be abbreviated to basis.

A brief explanation about the basis types is now in order. The basis type is a natural generalization of an equivalence relation which was discovered by Kaplansky and Mackey [7] on the class of $R$-modules of torsion-free rank 1. Define $f(R)$ to be the class of all sordinal (ordinal or $\infty$ ) valued functions on $R^{(k)}$ for all cordinals $k$. Let $f$, $g \in f(R)$. Define $f \sim g$ to mean both that Dom $f=\operatorname{Dom} g=R^{(k)}$ for some cordinal $k$ and that there is a matrix $\boldsymbol{\gamma}$ and a diagonal matrix $\delta$, both $k \times k$ invertible integral in $m(Q)$, such that $f(\alpha \gamma)=g(\alpha \delta)$ for every $\alpha \in R^{(k)}$. It is routine to show that $\sim$ is an equivalence relation 
on $f(R)$. Let $N$ be an $R$-module with $r(N)=k$. Then, each basis $\eta$ defines a function $g \in f(R)$ by $g(\alpha)=h_{p}(\alpha \eta)$ for every $\alpha \in R^{(k)}$. We can show that $g \sim g^{\prime}$ if $g^{\prime}$ is defined by another basis of $N$. The unique equivalence class of $f(R) / \sim$ determined by $N$ is called the basis type of $N$. Clearly, the basis type of $N$ is an invariant of $N$. The basis type of $N$ coincides with the Kaplansky and Mackey equivalence relation [7] if $r(N)=1$, with the Rotman strand of $N$ [10] if $r(N)<\omega$, and with the height equivalence of $N[1]$ if $r(N) \leqq \omega$.

3. Lemmas. We now initiate a series of lemmas that lead to the proof of our theorem.

Lemma 1. Two reduced $R$-modules $N$ and $N^{\prime}$ have the same basis type if and only if

(a) $r(N)=r\left(N^{\prime}\right)=k$, and

(b) for each pair of bases $\eta$ and $\eta^{\prime}$ of $N$ and $N^{\prime}$, respectively, there exists a matrix $\gamma$ and a diagonal matrix $\delta$, both $k \times k$ invertible integral matrices in $m(Q)$, such that the map $\rho$ defined by $\rho(\alpha \gamma \eta)=\alpha \delta \eta^{\prime}$ for every $\alpha \in R^{(k)}$ is a height-preserving isomorphism from the basic free submodule $[\gamma \eta]$ onto the basic free submodule $\left[\delta \eta^{\prime}\right]$.

This is an obvious generalization of Lemma 4.2 in [10]. Here, a free submodule of $N$ generated by a basis of $N$ is called a basic free submodule of $N$. Clearly, $\gamma \eta$ and $\delta \eta^{\prime}$ are bases of $N$ and $N^{\prime}$, respectively. Particularly, $\eta^{\prime}$ and $\delta \eta^{\prime}$ are stacked bases in the sense of [3] since $\delta$ is both diagonal and integral. Furthermore, it is easy to see that the condition (b) in the above lemma can be replaced by the following which gives a better picture of basis types.

(b') $N$ and $N^{\prime}$ contain basic free submodules $F$ and $F^{\prime}$, respectively, with a height-preserving isomorphism from $F$ onto $F^{\prime}$.

The following is our crucial lemma by which we can construct from a given basis a new one which will be suitable for our purpose. The notation $\pi_{B}$ denotes the coordinate projection from $A \oplus B \oplus C$ onto $B$.

Lemma 2. Let $N$ be a reduced $R$-module such that

(a) $N=A \oplus B \oplus C$,

(b) there are in $N$ disjoint subsets $\eta_{A}$ and $\eta_{B}$ such that $\eta_{A}$ and $\eta_{A} \cup \eta_{B}$ are bases of $A$ and $A \oplus B$, respectively, and

(c) if $x_{A} \in\left[\eta_{A}\right]$ and $x_{B} \in\left[\eta_{B}\right]$, then the height $h\left(x_{A}+x_{B}\right)$ is the minimum of $h\left(x_{A}\right)$ and $h\left(x_{B}\right)$.

Then, if we write $\eta_{B}=\left\{y_{i}: i<k\right\}$ where $k=\left|\eta_{B}\right|=r(B)$,

(d) there is in $m(Q)$ a $k \times k$ diagonal invertible integral matrix $\delta$ such that 
(e) $\tau=\pi_{B}\left(\delta \eta_{B}\right)$ is a basis of $B$, and

(f) there is a height-preserving isomorphism $\rho$ from the basis free submodule $\left[\delta \eta_{B}\right]$ onto the basic free submodule $[\tau]$ given by $\rho\left(\alpha \delta \eta_{B}\right)=\alpha \tau$ for every $\alpha \in R^{(k)}$.

Proof. Let $\zeta$ be a basis of $B$. Then, $\eta_{A} \cup \zeta$ is a basis of $A \oplus B$. Therefore, for each basis element $y_{i} \in \eta_{B}, i<k$, there is a nonzero element $d_{i} \in R$ with $d_{i} y_{i} \in\left[\eta_{A} \cup \zeta\right]$. Write $t_{i}=\pi_{B}\left(d_{i} y_{i}\right)$. Construct in an obvious way a $k \times k$ diagonal integral matrix $\delta$ with these $d_{i}$ 's and a $k$-dimensional column vector $\tau$ with these $t_{i}$ 's. It is clear that $\delta$ is invertible in $m(Q)$, and that $\tau$ is a basis of $B$. Thus, conditions (d) and (e) are proved. Since $\left[\delta \eta_{B}\right]$ and $[\tau]$ are free submodules of $N$, $\rho:\left[\delta \eta_{B}\right] \rightarrow[\tau]$, given by $\alpha \delta \eta_{B} \mapsto \alpha \tau$ for every $\alpha \in R^{(k)}$, is an onto isomorphism. Now

$$
h\left(\alpha \delta \eta_{B}\right)=h\left(\left(\alpha \delta \eta_{B}-\alpha \tau\right)+\alpha \tau\right) \leqq h(\alpha \tau)
$$

since $\alpha \delta \eta_{B}-\alpha \tau \in A$ and $\alpha \tau \in B$. On the other hand, by the condition (c),

$$
h(\alpha \tau)=h\left(-\left(\alpha \delta \eta_{B}-\alpha \tau\right)+\alpha \delta \eta_{B}\right) \leqq h\left(\alpha \delta \eta_{B}\right) .
$$

Hence, $h\left(\alpha \delta \eta_{B}\right)=h(\alpha \tau)=h\left(\rho\left(\alpha \delta \eta_{B}\right)\right)$, and $\rho$ is height-preserving. The proof is complete.

Recall that $M=\bigoplus\left\{M_{i}: i \in I\right\}$. Assume that $J \subseteq I$ and that $\eta$ and $\zeta$ are two bases of $M$. We define $M(J)=\bigoplus\left\{M_{i}: i \in J\right\}$, and $\eta(J)$ $=\eta \cap M(J)$. For each subset $\zeta_{0} \subseteq \zeta$, we define

$$
S_{M}\left(\zeta_{0}\right)=\bigcap\left\{L \subseteq I: \zeta_{0} \subseteq M(L)\right\}
$$

and $D \eta\left(\zeta_{0}\right)$ to be the minimal subset $\eta_{0} \subseteq \eta$ such that $\eta_{0} \cup\{z\}$ is linearly dependent for each $z \in \zeta_{0}$. A basis $\eta$ is said to be summandwise if each basis element $y_{j} \in \eta$ belongs to a single summand $M_{i(j)}$ (i.e., $y_{j} \in M_{i(j)}$ for each $\left.y_{j} \in \eta\right)$.

Lemma 3. Assume $M, M^{\prime} \in D(c, R, \omega)$ with direct decompositions $\left({ }^{*}\right)$. Let $r(M)=r\left(M^{\prime}\right)=k$, and let $\eta=\left\{y_{i}: i<k\right\}$ and $\eta^{\prime}=\left\{y_{i}^{\prime}: i<k\right\}$ be bases of $M$ and $M^{\prime}$, respectively, with $\eta^{\prime}$ summandwise. If $J \subseteq I$ is countable, then there is a set $T$ such that

(a) $T$ is countable and $J \subseteq T \subseteq I$,

(b) $\eta(T)$ and $\eta^{\prime}(T)$ are bases of $M(T)$ and $M^{\prime}(T)$, respectively, and

(c) $y_{i} \in \eta(T)$ if and only if $y_{i}^{\prime} \in \eta^{\prime}(T)$.

Proof. Let $\zeta$ be a summandwise basis of $M$. We construct for each $n<\omega$ a set $J_{n}$ such that

(1) $\left\{J_{n}: n<\omega\right\}$ is an ascending chain of countable subsets of $I$ with $J_{0}=J$, and 
(2) if we define, for each $n<\omega$,

$K_{n}=J_{n} \cup S_{M}\left\{y_{i} \in \eta: y_{i}^{\prime} \in \eta^{\prime}\left(J_{n}\right)\right\} \quad$ and $L_{n}=K_{n} \cup S_{M}\left(D_{\eta}\left(\zeta\left(K_{n}\right)\right)\right)$, then $J_{n+1}=L_{n} \cup S_{M^{\prime}}\left\{y_{i}^{\prime} \in \eta^{\prime}: y_{i} \in \eta\left(L_{n}\right)\right\}$.

Clearly, $T=\bigcup\left\{J_{n}: n<\omega\right\}$ satisfies the condition (a). Next suppose $y_{i} \in \eta(T)$. Then $y_{i} \in \eta\left(L_{n}\right)$ for some $n$. Hence, $y_{i}^{\prime} \in \eta^{\prime}\left(J_{n+1}\right) \subseteq \eta^{\prime}(T)$, and vice versa. Thus, the condition (c) is satisfied. To prove the condition (b), it suffices to show that the linearly independent subset $\eta(T)$ is maximal in $M(T)$ with respect to being linearly independent. Assume $y \in M(T)-M(T)_{t}$. Then, $y \in M\left(J_{n}\right)$ for some $n$, and hence $D_{\zeta}(y) \subseteq \zeta\left(K_{n}\right)$. Therefore, $D_{\eta}(y) \subseteq \eta\left(L_{n}\right) \subseteq \eta\left(J_{n+1}\right) \subseteq \eta(T)$, which implies that $\eta(T) \cup\{y\}$ is linearly dependent. Thus, $\eta(T)$ is a maximal linearly independent subset of $M(T)$. The proof is complete.

Assume that $N, N^{\prime} \in D(c, R, 0)$. We can write, for some cordinal $I$, $N=\bigoplus\left\{N_{i}: i \in I\right\}$ and $N^{\prime}=\bigoplus\left\{N_{i}^{\prime}: i \in I\right\}$. Then, the Kolettis theorem [5], [8], [9] tells that $N$ and $N^{\prime}$ have the same Ulm invariant if and only if there is a partition of $I$ into countable subsets $\left\{I_{j}\right\}$ such that $N\left(I_{j}\right)$ and $N^{\prime}\left(I_{j}\right)$ have the same Ulm invariant for each $j$ (consequently, $N \simeq N^{\prime}$ ). We next obtain the following lemma, which is an analog of the Kolettis theorem in the sense that our lemma concerns separation of the basis type, while his theorem that of Ulm invariant. We shall write $I^{i}$ for $U\left\{I_{j}: j<i\right\}$.

Lemma 4. Assume $M, M^{\prime} \in D(c, R, \omega)$ with direct decomposition (*). Then, $M$ and $M^{\prime}$ have the same basis type if and only if there is a partition of $I$ into countable subsets $\left\{I_{j}\right\}$ such that $M\left(I_{j}\right)$ and $M^{\prime}\left(I_{j}\right)$ have the same basis type for each $j$.

PROOF. The if-part is clear. For the only if-part, let $r(M)=r\left(M^{\prime}\right)$ $=k$. WLOG, we may assume that $I$ and $k$ are uncountable cordinals. By Lemma 1, $M$ and $M^{\prime}$ have bases $\eta$ and $\eta^{\prime}$, respectively, such that

(1) $\eta^{\prime}$ is summandwise, and

(2) the map $\rho$ from the basic free submodule $[\eta]$ onto the basic free submodule $\left[\eta^{\prime}\right]$ given by $\rho(\alpha \eta)=\alpha \eta^{\prime}$ for each $\alpha \in R^{(k)}$ is a heightpreserving isomorphism.

Let $r<I$ and suppose that for each $j<r$ there is a set $I_{j}$ such that

(3) $\left\{I_{j}: j<r\right\}$ is a family of pairwise disjoint countable subsets of $I$,

(4) $i \in I^{i+2}$ whenever $i+2 \leqq r$,

(5) $M\left(I_{j}\right)$ and $M^{\prime}\left(I_{j}^{\prime}\right)$ have the same basis type for each $j<r$,

(6) $\eta\left(I^{i}\right)$ and $\eta^{\prime}\left(I^{i}\right)$ are bases of $M\left(I^{i}\right)$ and $M^{\prime}\left(I^{i}\right)$, respectively, for each $i \leqq r$, and

(7) $y_{j} \in \eta\left(I^{i}\right)$ if and only if $y_{j}^{\prime} \in \eta^{\prime}\left(I^{i}\right)$ for each $i \leqq r$. 
Let us construct $I_{r}$ as follows. Let $J$ consist of the first element of $I-I^{r}$ and view this $J$ as the $J$ in Lemma 3. Then, Lemma 3 gives a countable set $T$ with $J \subseteq T \subset I$. Define $I_{r}=T-I^{r}$ and $\eta_{r}=\eta(T)-\eta\left(I^{r}\right)$. (It may happen that $\eta_{r}=\varnothing$.) It is easy to show that $\left\{I_{j}: j<r+1\right\}$ satisfies conditions (3), (4), (6) and (7) with $r+1$ in place of $r$. To prove the condition (5), we notice the following facts (8), (9), and (10).

(8) $M=M\left(I^{r}\right) \oplus M\left(I_{r}\right) \oplus M\left(I-I^{r+1}\right)$.

(9) $\eta\left(I^{r}\right)$ and $\eta\left(I^{r}\right) \cup \eta_{r}$ are bases of $M\left(I^{r}\right)$ and $M\left(I^{r}\right) \oplus M\left(I_{r}\right)$, respectively.

(10) If $x_{A} \in\left[\eta\left(I^{r}\right)\right]$ and $x_{B} \in\left[\eta_{r}\right]$, then

$$
h\left(x_{A}+x_{B}\right)=h\left(\rho^{-1}\left(x_{A}\right)+\rho^{-1}\left(x_{B}\right)\right)
$$

for $\rho$ is a height-preserving isomorphism.

It is easy from conditions (1) (2), (6) and (7) to see that $\rho^{-1}\left(x_{A}\right)$ $\in M^{\prime}\left(I^{r}\right)$ and $\rho^{-1}\left(x_{B}\right) \in M^{\prime}\left(I-I^{r}\right)$. Therefore,

$$
h\left(x_{A}+x_{B}\right)=\min \left\{h\left(\rho^{-1}\left(x_{A}\right)\right), h\left(\rho^{-1}\left(x_{B}\right)\right)\right\}=\min \left\{h\left(x_{A}\right), h\left(x_{B}\right)\right\} .
$$

Notice that the above conditions (8), (9), and (10) altogether constitute the hypothesis (a), (b) and (c) of Lemma 2. Now wellorder $\eta_{r}$ using the cordinal $k_{r}=r\left(M\left(I_{r}\right)\right)$. Then, by Lemma 2 , there is a $k_{r} \times k_{r}$ diagonal invertible integral matrix $\delta_{r} \in m(Q)$ and a basis $\tau_{r}$ of $M\left(I_{r}\right)$ with a height-preserving isomorphism $\rho_{r}$ from $\left[\delta_{r} \eta_{r}\right]$ onto $\left[\tau_{r}\right]$ given by $\rho_{r}\left(\alpha_{r} \delta_{r} \eta_{r}\right)=\alpha_{r} \tau_{r}$ for each $\alpha_{r} \in R^{\left(k_{r}\right)}$. Let $\eta_{r}^{\prime}=\left\{y_{i}^{\prime} \in \eta^{\prime}: y_{i} \in \eta_{r}\right\}$ and well-order $\eta_{r}^{\prime}$ precisely in the same way as $\eta_{r}$. By (b) of Lemma 3, and (1) and (6), $\eta_{r}^{\prime}$ is a basis of $M^{\prime}\left(I_{r}\right)$. Clearly, $h\left(\alpha_{r} \delta_{r} \eta_{r}^{\prime}\right)=h\left(\alpha_{r} \delta_{r} \eta_{r}\right)$ $=h\left(\alpha_{r} \tau_{r}\right)$ and hence, by Lemma $1, M\left(I_{r}\right)$ and $M^{\prime}\left(I_{r}\right)$ have the same basis type. Thus by transfinite induction, we can obtain the required partition $\left\{I_{j}: j<I\right\}$, and the proof is finished.

4. The proof of the theorem. The only if-part is self evident. For the if-part, assume that $M=\bigoplus\left\{M_{i}: i \in I\right\}$ and $M^{\prime}=\bigoplus\left\{M_{i}^{\prime}: i \in I\right\}$, with all summands $M_{i}, M_{i}^{\prime} \in(c, R, \omega)$, have the same Ulm invariant and the same basis type. Then, by the Kolettis theorem, we may assume that $M_{i}$ and $M_{i}^{\prime}$ have the same Ulm invariant for each $i \in I$. By Lemma 4, there is a partition of $I$ into countable subsets $\left\{I_{j}\right\}$ such that $M\left(I_{j}\right)$ and $M^{\prime}\left(I_{j}\right)$ have the same basis type (hence, the same height equivalence) for each $j$. Therefore, by Theorem $1, M\left(I_{j}\right)$ $\simeq M^{\prime}\left(I_{j}\right)$ for each $j$ and consequently $M \simeq M^{\prime}$. The proof is complete.

Recall the condition $\left(b^{\prime}\right)$ of Lemma 1 . The Ulm invariant determines the torsion part of $M$ and the basis type tells how a basic free 
submodule is located in $M$. The following obvious corollary gives a better picture of our theorem.

CoROLlaRy 1. $M \simeq M^{\prime}$ if and only if they have isomorphic torsion parts and they contain basic free submodules $F$ and $F^{\prime}$, respectively, with a height-preserving isomorphism from $F$ onto $F^{\prime}$.

Rotman and Yen [11] defined two $R$-modules $N$ and $N^{\prime}$ to be almost isomorphic in case there exist torsion $R$-modules $T$ and $T^{\prime}$ such that $N \oplus T \simeq N^{\prime} \oplus T^{\prime}$. It is easy to see that $N, N^{\prime} \in D(c, R, \omega)$ have the same basis type if and only if they are almost isomorphic. Thus, we have the following corollary which is Theorem $1^{\prime}$ in [11].

Corollary 2. Let $M, M^{\prime} \in D(c, R, \omega)$. Then $M \simeq M^{\prime}$ if and only if they have isomorphic torsion parts and they are almost isomorphic.

If we notice that the completeness of $R$ was needed nowhere except in Theorem 1, we can give the following which may be generalized to classes of modules over more general rings.

CoROLlaRy 3. Let $R$ be a discrete valuation ring (not necessarily complete) and assume that the Ulm invariants and basis types serve as a complete set of invariants for all countably generated reduced $R$-modules. Then the same result holds for all reduced direct sums of countably generated $R$-modules.

The author would like to acknowledge Professor Charles K. Megibben and the referee for valuable suggestions including the last corollary.

\section{REFERENCES}

1. C. Bang, Countably generated modules over complete discrete valuation rings, J. Algebra 14 (1970), 552-560.

2. - A classification of modules over complete discrete valuation rings, Bull. Amer. Math. Soc. 76 (1970), 380-383.

3. J. Cohen and H. Gluck, Stacked bases for modules over principal ideal domains, J. Algebra 14 (1970), 493-505.

4. L. Fuchs, Abelian groups, Akad. Kiad6, Budapest, 1958; republished by Internat. Series of Monographs on Pure and Appl. Math., Pergamon Press, New York, 1960. MR 21 \#5672; MR 22 \#2644.

5. P. Hill, Sums of countable primary groups, Proc. Amer. Math. Soc. 17 (1966), 1469-1470. MR 33 \#7408.

6. I. Kaplansky, Infinite abelian groups, Univ. of Michigan Press, Ann Arbor, Mich., 1954. MR 16, 444.

7. I. Kaplansky and G. W. Mackey, A generalization of Ulm's theorem, Summa Brasil. Math. 2 (1951), 195-202. MR 14, 128.

8. G. Kolettis, Jr., Direct sums of countable groups, Duke Math. J. 27 (1960), 111-125. MR $22 \# 1616$. 
9. F. Richman and E. Walker, Extending Ulm's theorem without group theory, Proc. Amer. Math. Soc. 21 (1969), 194-196. MR 39 \#1553.

10. J. J. Rotman, Mixed modules over valuation rings, Pacific J. Math. 10 (1960), 607-623. MR $22 \# 5632$.

11. J. J. Rotman and T. Yen, Modules over a complete discrete valuation ring, Trans. Amer. Math. Soc. 98 (1961), 242-254. MR 23 \#A227.

12. H. Ulm, Zur Theorie der abzahlbar-unendlichen Abelschen Gruppen, Math. Ann. 107 (1933), 774-803.

Emory University, Atranta, Georgia 30322 